\title{
Antecedentes al actual modelo de gobierno corporativo en los Estados Unidos de América: de 1934 a 2014
}

\author{
Dr. Iñigo Zavala \\ Universidad de Deusto
}

Sumario: I. Introducción. II. De la creación de la SEC (1934) a 1960. III. La trascendental década de los 70. IV. Los 80 y la desregulación. V. La continuidad en los 90. VI. La primera década del siglo, el escándalo «Enron» y la Sabarnes-Oxley Act.VII. Los últimos empeños regulatorios. VIII. Conclusiones.

Resumen: En el presente trabajo, realizamos un repaso a la historia del gobierno corporativo desde la creación de la SEC hasta nuestros días. Veremos cuán difícil ha sido para el pequeño accionista, hacer valer sus derechos, primero frente al todo poderoso management de las sociedades y luego frente a los inversores institucionales.

Palabras clave: accionista, gobierno corporativo, sociedad cotizada, mercado de valores, órgano de administración y comisiones.

Abstract: In this work we shall make a summary of the history of the corporate Governance, since the enactment of the SEC, till nowadays. We shall see the difficult path that the minority shareholder has got to follow, to the proper exercise of his rights, first against the power-full management of the company, and later to the institutional investors.

Keywords: Shareholder, corporate governance, public company, stock exchange, board of directors, commissions. 


\section{Introducción}

Este trabajo es un resumen del primer capítulo de la Tesis doctoral del autor, titulada «Gobierno corporativo de las sociedades cotizadas en los Estados Unidos: análisis crítico y retos pendientes». La selección de los contenidos se ha realizado, por mor de la mayor brevedad y la especificidad de esta publicación, dando prioridad a lo estrictamente relativo a la normativa aplicable al gobierno corporativo de las grandes corporations estadounidenses y teniendo en cuenta los tres tipos de normas que despliegan su eficacia sobre él: las que tienen su sede en el derecho de sociedades norteamericano; las que lo tienen en el New York Stock Exchange (NYSE) y en National Association of Securities Dealers Automated Quotation (NASDAQ); y, sobre todo, lasa que proceden de la Securities Exchange Commission (SEC), en virtud de la facultad delegada que, en el ámbito que nos ocupa, le otorgan tanto la Securities Act de 1933' ${ }^{1}$, como la Securities Exchange Act de $1934^{2}$.

Resulta ineludible una brevísima contextualización histórica y social. Así, recordamos que la utilización del tipo legal de la sociedad mercantil capitalista, con responsabilidad limitada de los socios a su aportación al capital, tiene su época de esplendor entre los años 1840 y 1850, como consecuencia de la necesidad de grandes capitales para financiar la construcción de los ferrocarriles en los EE.UU. Para entonces, la estructura de la sociedad por acciones (ya desde la East India ${ }^{3}$ ) tenía las características definitorias de la actual. La concentración empresarial que tuvo lugar a finales del siglo XIX dio lugar a la consolidación de la gran sociedad por acciones. Como dice Porter, tuvo un impacto determinante en la configuración del American way of life: «This did not happen universally or instantly, to be sure, but the corporation established its hegemony with relative speed and became the dominant institution in American society... The triumph of the corporation as the most powerful element in American life worked on many levels, affecting politics most immediately, reinforcing the prevailing gender, class, and ethnic order of American society, reshaping labor, and signaling the enshrinement of technology and science

1 SEC, http://www.sec.gov/about/laws/sa33.pdf.

2 SEC, http://www.sec.gov/about/laws/sea34.pdf.

3 Véase Alexander Schall para una aproximación a la historia de las sociedades y de la separación de poderes en ellas: "Corporate governance after the dead of the king. The origin of the separation of powers in companies», European Company and Financial Law Review, 8, 2011, 4, p. 477. 
as the new American gods and consumption as a way of life. In short order the corporation would restructure much of society according to its own image ${ }^{4} \gg$. Por otra parte, no es posible dar una completa visión de las fuerzas que desde distintos ángulos conformaron la historia del derecho de sociedades norteamericano -y por extensión, su actual sistema de gobierno corporativo - sin mencionar que desde el inicio se intentó mantener a los bancos fuera del negocio de la inversión en valores mobiliarios (es decir, limitando su actividad a la de meros bancos comerciales), con nulo éxito. Hubo que esperar a la Glass-Steagall Act ${ }^{5}$ — que obligaba a separar la actividad inversora de los bancos de la actividad comercial- para poder lograrlo. Para finalizar, mencionar que a pesar de los esfuerzos realizados por políticos de los dos principales partidos, con el objeto de que el Gobierno federal aprobase una ley general de sociedades, el resultado fue negativo. El Congreso rechazó siempre la idea de que la Administración federal pudiera aprobar normas sobre el gobierno y organización de las sociedades. Por el contrario, adoptó un enfoque más limitado «designed to introduce larger measures of integrity, not into corporate governance, but rather into the corporate securities distribution and trading processes ${ }^{6} \gg$.

\section{De la creación de la SEC (1934) a 1960}

Después del precedente párrafo introductorio podemos centrar nuestra atención en el proceso de recorte de derechos de los accionistas, en especial en lo relativo a las shareholders proposals, —realizado por la SEC, entre los años cincuenta y setenta- en cuanto que es un reflejo fiel de cuál era la postura mayoritaria de la doctrina y la administración, con respecto a aquellas materias en las que el gobierno corporativo de las sociedades comenzaba a manifestarse tímidamente.

Esta tendencia no es contradictoria con la apuesta por la shareho/der wealth maximization, en tanto que seguirían siendo los accionistas, los constituencies o interesados, en favor de los cuales tenía que gestionar la sociedad el management. Lo que cambia, en expresión de Bain-

4 Glenn Porter: The rise of the big business, Wheeling, Wiley-Blackwell, Illinois, 1973, 3. ${ }^{a}$ ed., p. 94.

5 The Internet Archives (organización sin ánimo de lucro) https://archive.org/details/ FullTextTheGlass-steagallActA.k.a.TheBankingActOf1933.

6 Alphonse A. SOMmeR: «The impact of the SEC on the corporate governance», Law and Contemporary Problems, 41, 1977, 3, p. 118. 
bridge ${ }^{7}$, son los «medios». Después de la Segunda Guerra Mundial, la corporation tenía que buscar como fin social el incremento del valor de la acción, pero utilizando como «medio» la gestión de los poderosísimos, especializados y clarividentes managers; en ellos debía residir el control último de la sociedad. Pero a partir de los setenta, la doctrina empezó a girar de nuevo (como consecuencia de la crisis del petróleo y de sonados escándalos de diversos directivos) para proponer que la shareholder wealth maximization se lograse utilizando como "medio» la shareholder primacy. Es decir, es el accionista en quién debe estar situado el verdadero control de la sociedad ya que nadie mejor que él sabe cuál es su beneficio. Se pasó, por lo tanto, de localizar el centro de control o poder de la corporation, del management al accionista.

Tradicionalmente, las leyes de los Estados regulaban el derecho de voto $^{8}$ de los accionistas ${ }^{9}$, qué materias (además del nombramiento de los consejeros) podrían ser objeto de votación en las juntas, y los procedimientos a seguir para emitir el voto. Aunque inicialmente el accionista tenía que asistir ${ }^{10}$ para votar a la junta, con el nacimiento de las grandes sociedades la delegación de voto pasó a ser la norma general. Con el objeto de restaurar la confianza del inversor en el mercado, la Securities Exchange Act de 1934 expresamente reguló en su sección catorce - esta vez sí, a nivel federal - los problemas que hasta entonces se asociaban con la delegación de voto con la finalidad de evitar los abusos denunciados por autores como Ripley, Berle y Douglas, durante los años anteriores.

Esta "sección catorce» no tenía la intención de "fortalecer» a los accionistas, sino solo de instigar la comunicación entre el management y los socios capitalistas, dentro del marco de la entonces vigente legisla-

7 Stephen Bainbridge: The new corporate governance in theory and practice, Oxford University Press, New York, 2008, p. 53. En donde realiza esta diferenciación, que ya encontrábamos en su artículo académico «Director primacy: the means and ends of corporate governance», Northwestern University Law Review, 97, 2003, p. 97 y que entendemos resulta acertada y clarificadora.

8 Véase Colleen A. DUNLAVY: "Social conceptions of the corporation: insights from the history of shareholder voting rights», Washington \& Lee Law Review, 63, 2006, p. 1347. La autora realiza un interesante recorrido por la evolución de la conceptualización del derecho de voto desde la época colonial hasta nuestro siglo. En concreto, analiza acertadamente la desviación del principio de "una acción, un voto» (aunque originariamente regía el principio de un voto por cabeza, con independencia de la aportación al capital) que tiene como efecto dos «radically different social conceptions of the corporation». Ibíd., p. 1387.

9 DALIA TSUK: "Shareholders as proxies: the contours of shareholder democracy», Washington \& Lee Law Review, 23, 2006, p. 1547.

10 lbídem, p. 1548. 
ción societaria de los estados (ibíd., 1546). Con este objetivo, de 1934 a 1942, la SEC aprobó una serie de "rules ${ }^{11}$ » que, perfeccionando la redacción de la mencionada "sección catorce», tenían como objetivo provocar el mencionado acercamiento y colaboración, incluso, entre los accionistas. Inicialmente muchas compañías se negaron a incluir en sus órdenes del día las propuestas que realizaban sus accionistas en las delegaciones de voto. Fue, entre otros muchos, el caso de la mastodóntica Bethlehem Steel Corporation, ante una propuesta de Lewis Gilbert ${ }^{12}$ — famoso activista de los derechos de los accionistas ${ }^{13}$ - , quién finalmente logró que la SEC obligara a la sociedad a retrasar la junta para incluir su propuesta en el orden del día ${ }^{14}$. Con motivo de estas negativas, la SEC en 1942, sugirió ciertos cambios en la "sección catorce» que además de tratar de garantizar el ejercicio de los derechos, pretendían proveer a los accionistas con los datos necesarios que les permitiesen votar de forma «informada».

La comunidad empresarial se opuso radicalmente a cualquier norma "of further legitiziming shareholders activism ${ }^{15}$ ». Ante esta negativa, la SEC finalmente aprobó una norma que obligaba a la dirección de la compañía a incluir las propuestas de los accionistas —en el orden del día con la solicitud de delegaciones para la junta- cuando estas fueran "proper subjects for action by the securitiesholders» (ibíd., 1551), así mismo, obligaba a hacer públicos los sueldos de sus directivos cuando estos superaran los 20.000\$ anuales. Garantizar el derecho individual de los accionistas a participar (aunque fuese de forma indirecta) en la junta general era un medio de proteger el ideal norteamericano de democracia (ibíd., 1553).

La siguiente modificación se realizó en 1947 y formalizaba la potestad de la SEC, de revisar las «shareholders proposals» que las sociedades querían excluir de su documentación por entender que no se

11 También denominadas «proxy rules», podrían ser el equivalente de las circulares de la CNMC.

12 Véase BRoc RoMANEK: "The pioneers of corporate governance», The Corporate Governance Advisor, 19, 2011, p. 1, en el que señala a los Gilbert —eran dos hermanos- como los precursores del gobierno corporativo, al liderar a los «Independently minded Shareholders».

13 Véase RICHARD MARENS: "Inventing corporate governance: the mid-century emergence of shareholder activism», Journal of Business and Management, 8, 2002, 4, p. 365. Sobre estos pioneros en el ejercicio de los derechos de los accionistas (también denominados «gadflies» o tábanos).

14 Tsuk, Shareholders as proxies, p. 1548

15 Es decir, una gestión en favor del accionista, pero sin contar con él. Similar a la máxima del Despotismo llustrado de las monarquías absolutas del siglo Xvill: «gobierno para el pueblo pero sin el pueblo». Ibíd., p. 1550. 
ajustaban a la norma de la «proper subjects for action by the securitiesholders ${ }^{16}{ }_{1}$.

En 1948 se aprobó otra rule que significó una nueva merma en los derechos de los accionistas: autorizó a las sociedades a excluir como «proper subject proposals» aquellas que afectasen a cualquiera de las tres siguientes situaciones: 1) las que tuvieran como objetivo una reclamación personal, o una queja contra la compañía o su dirección; 2) cuando la dirección hubiera incluido alguna propuesta del accionista en las dos últimas juntas, y este no hubiera acudido a las mismas, o no hubiera defendido su propuesta en las mismas; y 3) si una propuesta semejante hubiera sido presentada en la última junta, y no obtuvo el 3 por ciento de los votos ${ }^{17}$.

En los años posteriores, la SEC volvió a realizar dos importantes modificaciones: la primera, prohibir propuestas relacionadas con «promoting general economic, political, racial, religious, social or similar cau$\left.\operatorname{ses}^{18}\right\rangle$. El origen de este nuevo varapalo a los accionistas fue la solicitud realizada por un grupo antirracista The Congress of Racial Equality, a la Greyhound Corporation para que no separara en sus autobuses a los ciudadanos por razas $^{19}$. Y la segunda, que habilitaba al management «to omit any proposal wich impinges upon the duties and functions of the management ${ }^{20} »$, por lo tanto, se prohibían las relacionadas con los ordinary business. Como resultado de todo lo anterior, los accionistas ya solo podían centrarse en: la exactitud de los resultados y la política de dividendos. Es decir, las sociedades podían omitir, tanto las relacionadas con materias demasiado generales (políticas, raciales, religiosas, sociales), como aquellas que tuvieran relación con asuntos demasiado concretos (el propio negocio de la sociedad). Poco después, en una nueva vuelta de tuerca, se permitió a las sociedades excluir aquellas propuestas que aún siendo «proper proposals», hubiesen sido presentadas por lo menos una vez dentro de los tres años anteriores, y no hubiera obtenido al menos el 3 por ciento del voto. Si hubiese sido presentada dos veces en los tres años anteriores y no hubiera recibido el 6

16 Si la sociedad entendía que la propuesta que le enviaba el accionista no se ajustaba a la citada norma, la remitía a la SEC para que determinase su adecuación, o no, a la misma. En el caso de que la SEC confirmase el juicio de la sociedad, la propuesta no se incluía en el orden del día de la junta. Caso contrario, la sociedad tendría que incluirla.

17 Tsuk, Shareholders as proxies, p. 1555.

18 Ibídem.

19 «Peck contra Greyhound Corporation». 97F Supp.679, 679 (S.D.N.Y. 1951).

20 Seligman, The transformation, p. 271, citando las SEC minutes del 7 de agosto de 1953, 26 de enero de 1954, 19 de febrero de 1954, 2 de marzo de 1954 y 11 de marzo, de ese mismo año. 
por ciento de los votos; y si hubiese sido presentada tres veces y no hubiera obtenido el 10 por ciento de los votos. Así mismo, se exigía a los accionistas el envío de la propuesta sesenta días antes de la fecha en la que la documentación para solicitar la delegación de voto hubiera sido remitida por la sociedad el año anterior. Las posibilidades de éxito eran mínimas. En palabras de Tsuk: "Little was left of the original shareholder proposal rule...gradually..., the shareholders role as participant and the shareholder's role as investor converged. By the 1990s, this vision would dominate corporate $\operatorname{law}^{21} »$.

\section{La trascendental década de los 70}

Llegamos así a la década de los setenta, momento en el que el gobierno corporativo, en tanto que forma de organizar y alinear los intereses de los accionistas propietarios, los directivos y consejeros, concreta y define su actual significado 22 .

Tal como expone Seligman, en la década de los setenta se dieron las más importantes reformas en el gobierno corporativo de las sociedades cotizadas desde el New Deal: "For close of a decade, such fundamental questions as the legitimacy of the giant business corporation, the functions of the corporate board of directors, and the appropriate legal duties of corporate officers and directors were debated, first in public interest proxy contests and the technical literature, later before congressional and SEC hearings ${ }^{23} \gg$.

La guerra de Vietnam, la preocupación por la discriminación racial, el nacimiento de la conciencia medio ambiental, además del activismo en la defensa de otros derechos civiles, hizo que en 1970 se produjera una eclosión de propuestas sociales, a través de las proxy rules. Debido a la intensa presión social ${ }^{24}$ que se produjo durante estos años, la

21 Tsuk, Shareholders as proxies, p. 1560.

22 RANDALL K. MORCK, A history of corporate governance around the world: family business groups to professional managers, University of Chicago Press, Chicago, 2005, p. 5.

23 Joel Seligman, The transformation of Wall Street, Aspen Publishers, New York,

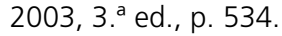

24 Esta presión social se materializó en una serie de campañas, entre las que podemos destacar la lanzada en 1968 por el Medical Committee for Human Rights, solicitando a la Dow Chemical que no fabricase más napalm con fines bélicos. Así mismo, el grupo liderado por el abogado activista Ralph Nader «Campaign GM», lanzó una campaña que tenía como objetivo persuadir a la General Motors para que mejorase su política laboral, medio ambiental y de transporte. Pero ninguna de estas actividades tenía una relación directa, ni afectaban a la estructura y mecanismos del gobierno corporativo. 
SEC en septiembre de 1972 modificó — de nuevo- la «sección catorce a-8», pero esta vez para abrir la puerta (aunque no suficientemente), a propuestas de accionistas que tuvieran como objetivo tratar cuestiones sociales, que solo podían ser excluidas por las sociedades si «was not significantly related to the business of the issuer or is not within the control of the issuer ${ }^{25}$ ».

Pero realmente el hecho que desencadenó la moderna concepción del gobierno corporativo de las sociedades, fue la quiebra de una importante compañía de ferrocarriles. La Penn Central ${ }^{26}$ era en el momento de su liquidación, la compañía de ferrocarriles más grande del país, así como la sexta corporación industrial. Durante los dos años anteriores a su colapso, el consejo de administración aprobó el abono de dividendos por importe de cien millones de dólares, mientras que la deuda de la sociedad crecía desbocadamente, por lo que la situación patrimonial sufrió un deterioro irreversible. La pasividad, negligencia, ignorancia y falta de compromiso del consejo de administración de esta sociedad, le valió el rechazo generalizado de la opinión pública.

La complacencia del consejo con el management fue explicada, entre otros, por el profesor de la Harvard Business School, Jonathan Macey 27 , por el hecho de que el nombramiento de nuevos consejeros estaba totalmente controlado por el management de la sociedad, y en especial por su Chief Executive Officer (CEO). En 1974 de las 6.615 solicitudes de delegación para el nombramiento de consejeros, supervisadas por la SEC, los consejeros con cargo en vigor fueron reelegidos, sin oposición, en 6.000 compañías (es decir el 90,6 por ciento) y en 6.606 (el 99,8 por ciento) la lista propuesta por el consejo resultó aprobada28.

25 SEC Annual Report de 1972, 14. La misma causó la SEC Act Release N. ${ }^{\circ} 9784$ de 22 de septiembre de 1972. SEC, http://www.sec.gov/about/annual_report/1972.pdf.

26 SELIGMAN, The transformation, p. 536.

27 Esto era una consecuencia directa de las decisiones judiciales (especialmente las del estado de Delaware), en las que se autorizaba a los miembros del consejo a pagar las campañas de reelección y nombramiento de nuevos miembros (por ellos propuestos), con cargo a los fondos sociales. Lo que significaba que todos aquellos accionistas que querían nombrar sus propios candidatos tenían que sufragarse la campaña; salvo en el improbable caso de que resultara vencedores en su intento. Tanto el número de accionistas en las grandes sociedades, como las distancias en aquel país-continente, hicieron que los costes de la elección de los consejeros a través de la delegación de voto no estuviese al alcance de cualquiera. Así que el nombramiento de consejeros por otros interesados distintos a los miembros del consejo con cargo vigente, resultó ser en la práctica una nueva quimera. Véase JonATHAN M. MACEY: Directors: myth and reality.

28 Michael E. MuRPhY: "The nominating process of corporate boards of directors: a decision making analysis», Berkeley Business Law Journal, 5, 2008, 2, p. 131. 
Así mismo, varios escándalos posteriores terminaron por hacer saltar todas las alarmas. A partir de 1974, la SEC tomó cartas en el asunto. Propuso que todas aquellas empresas que creyeran haber incurrido en alguna infracción de la normativa, realizaran una investigación interna al respecto y de forma voluntaria divulgasen su resultado. Más de 400 sociedades cotizadas publicaron un informe reconociendo haber realizado actividades cuestionables o incorrectas dentro del país o en el extranjero ${ }^{29}$. Nunca en la historia de la SEC, tales escándalos (Penn Station, sobornos y contribuciones ilegales a partidos políticos), obtuvieron tan poca reacción ${ }^{30}$.

En 1976 la SEC consiguió que el NYSE exigiera a todas las compañías que cotizaban en la bolsa de Nueva York, el cumplimiento de una serie de requisitos relativos a medidas de gobierno corporativo, relacionadas con el consejo de administración como el nombramiento de consejeros independientes ${ }^{31}$, y a la creación de un comité de auditoría ${ }^{32}$.

Históricamente ha sido a través de de los settlements ${ }^{33}$, la vía por la que la SEC ha realizado sus más notables incursiones en el mundo del gobierno corporativo (hasta la promulgación de la Sox). Básicamente, los acuerdos de la Comisión —en esta etapa - relacionados con el gobierno corporativo, se pueden agrupar en torno a las siguientes materias: 1) acuerdos que exigían el nombramiento de más consejeros independientes («SEC contra Canadian Javelin», SEC Litigation Release N. ${ }^{\circ} 6.441$ (S.D.N.Y. 1974); 2) acuerdos de naturaleza especial para una circunstancia determinada, o bien que de forma continua son impuestos a un consejo ya existente ( $\mathrm{SEC}$ contra Emerson Ltd.», Litigation Release N. ${ }^{\circ} 7.392$ (D.D.C. 1976); 3) acuerdos que requieren el nombramiento de un auditor y/o de algún comité del consejo ( SEC contra Mattel Inc.», SEC Litigation Release N. ${ }^{\circ} 6.531$ y 6.532 (D.D.C. 1974); 4)

29 MuRPHY, The nominating process, p. 134.

30 SELIGMAN, The transformation, p. 551.

31 JefFREY N. GORDON, "The rise of independent directors in the United States, 19502005: of shareholder value and stock market prices», Stanford Law Review, 89, 2007, pp. 1477-9. Según este autor, el término «consejero independiente», se incorporó al léxico del gobierno corporativo en la década de los setenta, ya que hasta entonces la división era entre consejeros externos e internos, lo que no quedó resuelto en ese momento, fue el significado del término «independiente».

32 SOMMER, The impact of the SEC, p. 130.

33 Acuerdos alcanzados entre la SEC y la empresa infractora que evitan el inicio y/o continuación del expediente administrativo sancionador. Véase nota de prensa emitida por la SEC en la que hace público el settlement alcanzado con J.P. Morgan, en virtud del cual esta se obliga a pagar una multa de 980 millones de dólares, y reconoce el incumplimiento de la legislación federal (en concreto la Sox); SEC, http://www.sec.gov/News/ PressRelease/Detail/PressRelease/1370539819965. 
acuerdos que implican el nombramiento de un asesor especial que dirija una investigación sobre ciertas prácticas de la empresa señaladas por la SEC. ( SEC contra General Tire and Rubber Co.», SEC Litigation Release N. ${ }^{\circ} 7386$ (D.D.C. 1974), y 5) el nombramiento de un auditor especial que apoye el trabajo de un asesor especial ( SEC contra Mattel Inc.», SEC Litigation Release N. ${ }^{\circ} 6531$ y 6532 (D.D.C. 1974). Obviamente estos «acuerdos» entre la SEC y la sociedad denunciada, solo afectaba a las partes implicadas, pero no obstante, y tal y como antes hemos dicho, tenían una influencia importante sobre todas las empresas cotizadas ${ }^{34}$.

Aunque en su origen la SEC era la agencia gubernamental con las facultades necesarias para el estudio de las posibles necesidades de reforma del gobierno corporativo, esta competencia era considerada accesoria o tangencial a sus responsabilidades principales que eran: la regulación de los mercados, la de procurar adecuada información sobre las sociedades cotizadas, y la persecución del fraude, primero a nivel administrativo y luego judicial ${ }^{35}$. Realmente, la idea de la regulación del gobierno corporativo de las sociedades era considerada como una cuestión secundaria, ligada al problema de la información. Ello provocó que la respuesta de la SEC a este debate fuera un tanto paradójica. En efecto, por un lado, la agresiva campaña lanzada por la Comisión, al objeto de que se informasen y publicitasen los distintos casos de sobornos en los que se vieron implicadas importantes empresas en aquella década; y por otro lado, que la respuesta dada directamente al problema del gobierno corporativo fue realmente limitada. En 1978, la SEC adoptó una rule ${ }^{36}$ que a lo único que obli-

34 SOMMER, The impact of the SEC, p. 130.

35 Véase SEC, http://www.sec.gov/news/speech/1962/113062conwill.pdf. El discurso del comisionado Allan F. Conwill en Tulsa Oklahoma en 1962, titulado "Current functions and objetives of the Securities Exchange commission», en el que de forma breve y didáctica se recogen estos tres objetivos. Así mismo, la actual presidente de la SEC, Mary Jo White, afirma, "our core mission of protecting investors, promoting fair, orderly and efficient markets and facilitating capital formation». Fiscal Year 2013 Agency Financial Report, 2. SEC, https://www.sec.gov/about/secpar/ secafr2013.pdf.

36 Véase Robert BaldWin, Martin CaVe y Martin LodGe, Understanding regulation. Theory, strategy, and practice, Oxford University Press, New York, 2012, 2. a ed., p. 230. «Rule» es como se denomina a las normas que, sin rango de Ley, aprueba la SEC en ejercicio de la capacidad reglamentaria que le otorga, en especial la Securities Exchange Act de 1934; con el objeto de ordenar, fiscalizar y velar por el correcto funcionamiento del mercado de valores. Véase SEC.www.sec.gov/whatwedo/shtml, en donde se da una somera información sobre el proceso administrativo que desemboca en la aprobación de una rule por parte de la SEC. 
gaba a las empresas cotizadas era a hacer pública información adicional (no a la toma de acuerdos en un sentido determinado) sobre: la independencia de sus consejeros; si las sociedades tenían comités de auditoría, nombramientos y retribuciones; y si algunos de los consejeros habían acudido a menos del 75 por ciento de las reuniones del consejo el año anterior.

En los años siguientes, diversas organizaciones no gubernamentales de marcado carácter profesional entraron en juego realizando distintas propuestas relacionadas con la materia. En 1980, y copatrocinada por la American Bar Association y el NYSE, la American Law Institute (ALI) convocó una conferencia con el fin de dar respuesta a los acontecimientos que en materia de gobierno corporativo habían tenido lugar desde 1970. Dado el prestigio de ALI, se pensó que sus conclusiones podrían incluso determinar una acción por parte del legislativo. Efectivamente, una impresionante refriega político-jurídica fue el resultado de tales propuestas, que coincidieron con el cambio de inquilino en la Casa Blanca tras la llegada de Ronald Reagan, en 1980, a la presidencia del país.

Es en esta década y a raíz de estos estudios y propuestas cuando se empieza a conformar el consejo de administración como un «monitoring board», conceptualización que ha perdurado hasta nuestros días, tal y como con todo vigor defiende Bainbridge. Pero realmente, desde un punto de vista teórico, el espaldarazo definitivo a esta concepción se fraguó en el trabajo del profesor de Berkeley, Melvin Einserberg: "The structure of the corporation» que, en palabras de Tsuk es: «perhaps the most important work on Corporate Law since Berle $\&$ Mean's The modern corporation and private property ${ }^{37}$ ». Eisenberg concluyó que la más importante función del consejo era nombrar, y en su caso separar, al CEO, y controlar el funcionamiento del los altos directivos. Escribió, «under the monitory model...the role of the board is to hold the executives accountable for adequate results, while the role of the executives is to determine how to achieve such results $^{38}$ ». En 1980, ya nadie ponía en duda que la función de control era la apropiada para el consejo. Esta idea fue reafirmada con la publicación en 1982 del Tentative Draft del (ALI) «Principles of corporate governance» (cuya versión final fue publicada, tal y como ya hemos dicho en 1992, tras una década de luchas), cuyo autor principal,

37 DALIA TSUK: "Status bound: the $20^{\text {th }}$ century evolution of directors liability», New York University Journal of Law and Business, 5, 2012, p. 135.

38 Melvin A. EISENBERG: The structure of the corporation. A legal analysis, Beard Books, Washigton, 1976, p. 165. 
como señala Bainbridge ${ }^{39}$, fue Eisenberg. Consecuentemente, acabó de tomar forma la posición de que los deberes de los consejeros eran para con los accionistas exclusivamente.

Otro hecho de gran trascendencia en la configuración del actual sistema de corporate governance en los EE.UU, aunque inicialmente nadie pensó en sus consecuencias, fue la adopción por la SEC el 23 de enero de 1975, de la rule 19b-3 que liberalizaba las comisiones que las distintas agencias y brokers cobraban por la prestación de sus servicios y que desde el pacto de Buttonwood Tree ${ }^{40}$, eran iguales para todos. Esta norma entro en vigor el 1 de mayo del mismo año, por lo que popularmente es conocida por «May Day». La aprobación de esta medida significó la bajada de las comisiones hasta entonces pactadas, en casi dos terceras partes, ocasionando que los inversores institucionales (y también los particulares), incrementaran su actividad ${ }^{41}$. El flujo de dinero que supuso la progresiva liberalización del movimiento de capitales a nivel mundial, provocó que las autoridades tomasen medidas para hacer sus mercados más atractivos a los inversores de otros estados. Esto se concretó en una apuesta importante por la modernización de los sistemas informáticos de contratación, así como por la reducción del coste de las operaciones en el mercado. Y debido a que el volumen contratado es inversamente proporcional al coste de transacción, la rotación en los mercados se disparó, por lo que a su vez la media temporal de tenencia de las acciones se redujo de forma exponencial ${ }^{42}$.

\section{Los 80 y la desregulación}

El nombramiento de Ronald Reagan como presidente del país al inicio de la década de los ochenta, además de significar una nueva

39 BAINBRIDGE, Corporate governance, pp. 53-7.

40 Acuerdo firmado el 17 de mayo de 1792 entre veinticuatro brokers, según el cual el corretaje a cobrar por las operaciones de compra y venta de acciones en el NYSE, se realizaría aplicando una comisión fija sobre la suma intermediada: "We the Subscribers, Brokers for the Purchase and Sale of the Public Stock, do hereby solemnly promise and pledge ourselves to each other, that we will not buy or sell from this day for any person whatsoever, any kind of Public Stock, at a less rate than one quarter percent Commission on the Specie value and that we will give preference to each other in our Negotiations. In Testimony where of we have set our hands this 17th day of May at New York, 1792». NYSE, http://www.nyse.com/about/history/timeline_events.html.

41 Pavlos E. Masouros: Corporate law and economic stagnation. How shareholders value and short-termism contribute to the decline of the western Europe, Eleven International Publishing, The Haghe, 2013, p. 81.

42 Ibíd., p. 87. 
mayoría política en el Congreso, supuso el nombramiento de un nuevo presidente de la SEC, Jhon Shad ${ }^{43}$, y un importante impulso desrregulatorio. Efectivamente, cuando el ALI publicó en 1982 su Tentative Draft número uno ), el mundo político, jurídico y financiero en general, reaccionó con pánico. Los cambios de personas (la nueva mayoría republicana sustituyó a los antiguos representantes de la administración demócrata) operados en los consejos y juntas directivas de las distintas sociedades, asociaciones y organismos de toda clase que, inicialmente habían respaldado la actuación de la ALI, hicieron que todas ellas retiraran su apoyo al mencionado e inicial Tentative Draft. Así mismo, tanto el poderoso lobby de los managers y directivos, como el mundo de los negocios en general, apreciaron en estas propuestas un peligro para sus intereses económicos. Este borrador proponía dotar a los accionistas con los derechos suficientes para poder realizar una eficaz labor de control de los directivos y, de esta forma, impedir que utilizasen la sociedad en beneficio propio. Este argumento exigía por lo tanto, un refuerzo de la presión reguladora para luchar contra la supuesta indefensión de los (pequeños) accionistas. En resumen: empieza a cambiar el centro de poder del management a los shareholders, nos encontramos con los primeros balbuceos de la shareholder primacy (insistimos, minado el prestigio de los managers por escándalos mayúsculos y retornos económicos que ya no tenían nada que ver con los de antaño).

Al objeto de aplacar las críticas, la dirección de la ALI decidió sustituir a los responsables de la redacción del primer documento, por otros expertos más en sintonía con la corriente desrreguladora existente en el país. Fruto de este cambio, fue la modificación del primer borrador, y la alteración de la propuesta inicial que sugería que las recomendaciones contenidas en el mismo deberían ser de obligatorio cumplimiento (principalmente a través de la acción de la SEC); por una propuesta de perfil mucho más bajo, en el sentido de transformar la obligatoriedad inicial de sus conclusiones por recomendaciones de voluntario cumplimiento. Y así, finalmente, en 1992 la ALI aprobó sus «Principles of corporate governance: analysis and recomendations ${ }^{44} \gg$; siendo publicadas en 1994 con una redacción tan descafeinada que ni siquiera fue objeto de censura alguna por sus iniciales detractores.

43 Que anteriormente había sido presidente de una conocida agencia de valores en Wall Street. New York Times,http://www.nytimes.com/1994/07/09/obituaries/john-s-rshad-dies-at-71-sec-chairman-in-the-80-s.html.

${ }_{44} \mathrm{ALI}$, http://www.ali.org/index.cfm?fuseaction=publications.ppage\&node_id=88. 
La doctrina crítica ${ }^{45}$ con la base intelectual que cimentó el primer y fallido borrador de la ALI, siguió defendiendo la postura de que no era demasiado preocupante que los directivos confundiesen la prosperidad de la sociedad con la suya propia. Y ello era debido a que el mercado contaba con otros mecanismos para alinear los intereses de los shareholders y de los managers. Estos mecanismos fueron, durante la década de los ochenta, principalmente el mercado de OPAS, y de adquisiciones. Durante estos años, se desarrollaron una serie de instrumentos legales y financieros que propiciaron la posibilidad de que todas aquellas empresas que estuvieran infravaloradas, debido a la falta de acierto en la gestión de sus directivos, pudiesen ser objeto de una OPA ${ }^{46}$. El éxito de esta significaría la salida del antiguo equipo directivo y su sustitución por otro de la confianza del nuevo accionista mayoritario. Esta amenaza, prosiguen los defensores de estos mecanismos, es un factor suficiente para disuadir a los directivos de las empresas cotizadas de descuidar sus obligaciones o «confundir» los intereses propios con los sociales. En definitiva, el mayor precio que sobre el valor de la acción pagaba el ofertante, se entendía que podía recuperarlo sustituyendo a los directivos por otro equipo más capacitado que, obviamente, mejoraría los resultados.

Pero este mercado realmente frenético (y casi inexistente en Europa y Japón) llegó a su fin cuando las poderosas asociaciones de di-

45 JonAthan R. MACEY: "The transformation of the american law institute», George Washington Law Review, 61, 1993, p. 1212.

46 Véase, dentro de la abundantísima literatura al respecto generada en aquellos años: Michael C. JenSEN: «Takeovers: their causes and consecuences», Journal of economic Perspectives, 21, 1988; YACOB AMIHUD Y BARUCH LeV: «Risk reduction as a managerial motive for conglomérate mergers», Bell Journal of Economics, 12, 1981; RlCHARD RolL: "The hubris hypothesis of corporate takeovers», Journal of Business, 59, 1986, tratando de explicar las consecuancias negativas que estas operaciones pueden generar a los adquirientes; ROBERTA ROMANO: "A guide to takeovers: theory, evidence, and regulation», Yale Journal on Regulation, 9, 1992; O desde el punto de vista del origen fiscal de algunas de estas ofertas: Roland J. GILSON, Myron S. SCHOles y Mark A. WolfSON, "Taxation and the dynamics of corporate control: the uncertain case for tax motivated acquisitions», en JOHN COFFEe, LOUIS LOWENSTEIN y SUSAN ROSE-ACKERMAN, Knights, riders, and targets: the impact of hostile takeovers, Oxford University Press, New York:, 1988; Alan AuerbaCh y David ReISHus: «The impact of taxation on mergers and acquisitions», en Alan AUerBACH, Mergers and acquisitions, University of Chicago Press, Chicago, 1988. También desde la perspectiva de las management buyout: STEVEN N. KAPLAN: "Managements buyouts: evidence on taxes as a source of value», Journal of Finance, 44, 1989; KATHERINE SCHIPPER y ABBIE SMITH, "Effects of management buyouts on corporate interest and depreciation tax reductions», Journal of Law and Economics, 34, 1991. 
rectivos ${ }^{47}$, presionaron a los legislativos de los Estados federados para que modificaran la ley y de esta forma entorpecieran el posible éxito de las OPAS. Además, la modificación de los estatutos de muchas sociedades para incorporar las denominadas poison pills (cláusulas estatutarias que hacen prácticamente imposible el control de la sociedad a quien lo adquiera a través de una OPA ${ }^{48}$ ), y el endurecimiento de las condiciones financieras, hizo que esta forma de control también se diluyese.

Ante esta situación, parecía que el manager-centric model, volvía a imponerse, y que los derechos de los accionistas - ante su desidia e inoperancia - quedaban relegados a un mero reconocimiento formal, vacíos de contenido. Incluso el reputado profesor de la Universidad de Yale, Schwartz, firme defensor de los derechos de los accionistas, escribió, "that shareholder participation is not capable of working well because of its impracticability and because of the rational indifference of shareholders to participation in corporation affairs $49 »$.

Durante esta década, la SEC, presidida John Shad, quien, por formación (profundamente influenciado por la Teoría del Análisis Económico del Derecho), y por convicción política, era partidario de la acción auto-correctora del mercado ${ }^{50}$, limitó su respuesta a las «hostile tender offers» que caracterizaron estos años, a la remisión al mercado y a la legislación de los Estados, lo que acumulativamente hizo que se reforzara la posición del management de las sociedades opadas u objeto de oferta, con el resultado de la desactivación de aquellas tal y como acabamos de exponer. Su sucesor en el cargo - Ruder, en 1987- ya anticipó que esta clara inclinación en favor de los managers, y las consiguientes modificaciones estatutarias que tuvieron como resultado la admisión generalizada de las poisons pills, sin duda dificultarían la transmisión de las acciones.

Podemos afirmar, por lo tanto, que en esta década muy poco quedó del ideal del principio de siglo xx de la democracia participativa (o shareholder democracy) de los accionistas (también es verdad que las estructuras accionariales y tipología de los mismos habían cambiado totalmente). Con la eliminación de cualquier otra alternativa

47 Jonathan R. MACEY, Corporate governance, Princeton University Press, Princeton, New Jersey, 2008, p. 122.

48 Véase Frankuin A. Gervutz: Corporation law, West, Minnesota, 2010, p. 706.

49 DonAlD SCHWARTZ: "Shareholder democracy: a reality or a chimera?», California Management Review, 25, 1983, p. 55.

50 SELIGMAN, The transformation, p. 576. 
para influenciar a la dirección de la compañía, los accionistas quedaban avocados al ejercicio de sus derechos "del mercado» de "exit» o «voice ${ }^{51} »$. El accionista individual era un inversor con poco, si es que algún poder, y su participación en la gestión de los asuntos sociales se volvió una reliquia de tiempos pasados. Solo la pujante llegada de los inversores institucionales logró hacer despertar en el comunidad académica, la financiera y la clase política, un interés que, hábilmente utilizado por aquellos (bajo el ropaje de un supuesto y renacido interés por los derechos de los accionistas), sirvió para transformar, en provecho propio, una reacción, que, tal y como veremos, ha llevado a destinos no deseados.

\section{La continuidad de los 90}

En la década de los noventa, con la llegada de Clinton a la casa Blanca, Levitt fue nombrado presidente de la SEC, y estuvo en el cargo desde 1993 hasta 2001. Trató de resistirse a la inercia desreguladora que, a pesar de la llegada de los demócratas al poder, aún prevalecía tanto en la comunidad política como en la académica. De hecho, durante su mandato, se aprobaron algunas de las leyes más neoliberales de la historia de los Estados Unidos. Como ejemplo, señalar que nada más tomar posesión del cargo, la «Task Force on Disclosure Simplification» recomendó la eliminación de ochenta y un rules de la Comisión, veintidós formularios, así como la modificación de decenas de otros ${ }^{52}$. Si bien es cierto que la esencia del sistema de información quedó inalterada.

Así mismo, se aprobaron: la Private Securities Litigation Reform Act (1995) que limitó y redujo de forma drástica la posibilidad de demandar a las sociedades y sus administradores por daños causados a sus accionistas (realmente puso un poco de orden en una legislación dispersa y com-

51 También llamada «The Wall Street rule». Con esta expresión se hace referencia a que los accionistas tienen dos opciones para mostrar su desacuerdo con la gestión de la sociedad que realiza su dirección, bien salirse (exit) es decir, vender sus acciones; bien quedarse, y ejercitar su derecho a ser oídos en la junta (voice). Véase AlBerT O. HIRSCHMAN, Voice and loyalty: responses to decline in firma, organizations and states, Harvard University Press, Cambridge, Massachusetts, 1970. En IRIS H-Y. CHIU, The foundations and anatomy of shareholder activism, Hart Publishing, Portland, Oregon, 2010, p. 38.

52 SEC, Task Force report on Disclosure Simplification, 1995-96. Fed. Sec. L. Rep. (CCH).85, 738 (Mar. 3, 1996). En SELIGMAN, The transformation, p. 633. 
plicada que generaba cierta inseguridad jurídica53); la National Securities Markets Improvement Act (1996) y la Gramm-Leach-Bliley Act ${ }^{54}$ (1999), ratificada por el presidente Clinton, y que de hecho supuso el fin de la separación entre bancos industriales y comerciales, en vigor desde la Glass-Steagall Act (1933). Por el contrario, es justo señalar que su oposición, en julio de 1995 a la propuesta del senador Jack Fields denominada Proposal for a Capitals Market Deregulation and Liberalization Act ${ }^{55}$ (recordar que desde 1994 los republicanos tenían la mayoría en ambas cámaras) fue esencial a la hora de conseguir abortar la misma.

Pero, si por algo se recordará a Levitt, será por su determinación en reclamar un endurecimiento de las normas sobre auditoría y contabilidad de las sociedades. Persiguió - y logró- que el auditor interno pasara a ser parte del sistema de gobierno corporativo de la sociedad. Su falta de confianza en estos profesionales, le hizo promover una serie de medidas encaminadas al cumplimiento estricto de la normativa contable (especialmente relativos a la contabilización e información de derivados financieros, así como a evitar el prematuro reconocimiento de ingresos). Como consecuencia, consiguió convencer a los presidentes del NYSE y del NASDAQ, para que nombraran un grupo de expertos que analizara las funciones y efectividad de los comités de auditoría que los consejos de administración de las sociedades cotizadas tenían obligación de nombrar. El grupo estaría presidido por J.C. Whitehead (expresidente de Goldman Sach) e Ira Milstein, socia de una importante firma de abogados ${ }^{56}$. Este comité se denominó Blue Ribbon Committee on Improving the Effectiveness of Corporate Audit Committees. En 1999

53 The PSLRA was enacted in order to minimize the practice of certain law firms that commenced class actions against companies alleging wrongdoings in general terms and then proceed on «fishing expeditions» to attempt to uncover malfeasance on the part of companies. The very liberal discovery rules in litigation in the United States generally permits litigants to engage in extensive pretrial discovery proceedings thereby forcing companies to open their books and records. Rather than engage in such costly litigation, companies often settle for millions of dollars, with that law firms that commenced the action receiving sizeable fees. Congress addressed the alleged abuses by the enactment of PSLRA. ROY GIRASA: Corporate governance \& finance law, Pelgrave-MacMillan, New York, 2013, p. 205.

54 Esta ley ha sido señalada por la comunidad académica y profesional, como una de las causantes de la crisis financiera de 2008. De hecho, su máximo mentor y presidente del Senate Banking Committee de 1995 al 2000, fue acusado por la revista Time, de ser una de las veinticinco personas causantes de la crisis. Time: http://www.time. com/time/specials/packages/article/0,28804,1877351_1877350_1877330,00.html.

55 SELIGMAN, The transformation, p. 674. En palabras de este autor, el congresista Fields, presidente del Subcomité de Telecomunicaciones y Finanzas del Congreso, pretendía «the most sweeping reform of securities laws in the last 40 years».

56 lbíd., p. 720. 
publicó sus conclusiones con diez recomendaciones, la principal, que toda compañía que cotizase en el NYSE o en el NASDAQ, y con una capitalización superior a los veinte millones de dólares, debería tener un comité de auditoría compuesto íntegramente por consejeros independientes, con un mínimo de tres miembros, cada uno de los cuales debería tener amplios conocimientos de contabilidad. Además, el Comité recomendó:

that the NYSE, and the NASD require the audit committee of each listed company to (i) adopt a formal written charter that is approved by the full board of directors and that specifies the scope of the committee's responsibilities, and how it carries out those responsibilities, including structure, processes, and membership requirements, and (ii) review and reassess the adequacy of the audit committee charter on an annual basis»; that the revised audit committee be «Responsive for ensuring the receipt from the outside auditors of a formal written statement delineating all relationships between the auditor and the company, consistent with Independent Standards Board Standards 57 .

Lógicamente, estas recomendaciones fueron adoptadas por la SEC, el NYSE y el NASDAQ. La aprobación de estas normas coincidió además con la investigación realizada por la Comisión sobre una serie de infracciones cometidas por Arthur Andersen y Coopers \& Lybrans (luego fusionada con Pricewaterhouse) en la que de forma increíble, se descubrió que durante el período investigado esta última había infringido la norma que regulaba la prestación de los servicios de auditoría en más de 8.000 ocasiones. Esto hizo manifestar a Lewitt, "the lesson of this episode is cristal clear: self-regulation by the accounting profession is a bad joke... The firms would never subject themselves to scrutiny unless forced to do $\mathrm{so}^{58}$ ».

La siguiente revisión que realizó la SEC sobre las normas de independencia de los auditores, se focalizó en el control de los servicios - distintos a los de auditoría- que los auditores prestaban en paralelo con los propios de su profesión (es decir, asesoramiento legal, consultoría, prestación de servicios informáticos, etc.). Tras una impresionante guerra entre las (entonces) «big five» y la SEC, esta aprobó59 en noviembre de 2000, la Revised Accounting Independence Rule60.

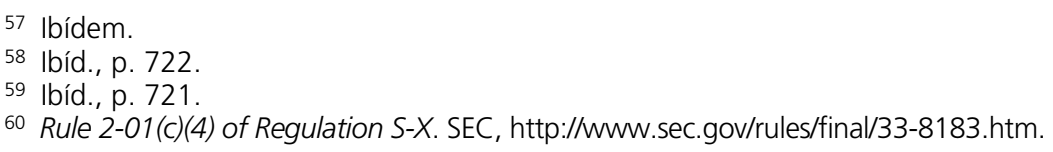


También al inicio de esta década de los noventa se produce la explosión a nivel internacional del estudio del gobierno corporativo, siendo Reino Unido el camino de entrada a nuestro continente. El primer documento «oficial» fue el «Informe Cadbury ${ }^{61}$ », que marcó un antes y un después en la historia europea sobre esta materia. El citado informe se elaboró en un momento de crisis económica e incluso existencial del Reino Unido, ante la comprobación de la pérdida evidente de su liderazgo político y social a nivel mundial. Así mismo, coincidió con una serie de escándalos mayúsculos ${ }^{62}$ que se achacaron a la falta de un apropiado gobierno corporativo. Todo ello, junto con la habilidad del Comité para lograr que el London Stock Exchange ${ }^{63}$ lo incluyese como un anexo a las normas que debían cumplir las compañías cotizadas en este mercado, le otorgó una influencia sobre la mayoría de los códigos posteriores de todo el continente que, aún hoy, es incuestionable.

Esto coincidió en el tiempo con que en toda Europa se puso en marcha una política liberalizadora de los mercados de capitales, así como privatizadora de muchas de las grandes empresas estatales, facilitando la rápida adopción de códigos de buen gobierno. Finalmente -y como consecuencia de todo lo anterior- se produjo la llegada de los grandes fondos americanos al capital de las grandes sociedades europeas. Estos fondos, antes de entrar en el accionariado de cualquier sociedad, ponían como condición sine qua non, que esta tuviese aprobadas unas elementales normas de corporate governance.

En 1999, con la aprobación y publicación de los «principios de gobierno corporativo» por parte de la OCDE64, se produjo el espaldarazo definitivo al estudio, aplicación e implantación de los denominados «códigos de buen gobierno» que tanta expansión (no nos atrevemos a decir que también éxito, visto lo visto) tuvieron por todo el planeta. Fueron diversos los estudios de consultoras (en este caso, Mckinsey ${ }^{65}$ ) y

61 European Corporate Governance Institute (ECGI), http://www.ecgi.org/codes/documents/cadbury.pdf.

62 En especial lan Robert Maxwell, magnate de los medios de comunicación británicos, quien utilizó - y vació- los fondos de pensiones de los empleados del Mirror Group. Véase New York Times, 12 octubre 1991, http://www.nytimes.com/1991/12/10/ business/worldbusiness/10iht-max_2.html.

63 Véase London Stock Exchange. http://www.Iondonstockexchange.com/home/ homepage.htm.

64 OECD, http://www.oecd.org/corporate/ca/corporategovernanceprinciples/315577 24.pdf. Estos principios fueron actualizados en el año 2004.

65 Mckinsey and Company http://www.mckinseyquarterly.com/Governance/Boards/ Three_surveys_on_corporate_governance_965. 
analistas que aseguraban que los inversores institucionales estaban dispuestos a pagar sobreprecios de hasta un 30 por ciento por las acciones de sociedades que cumpliesen estrictamente con las exigencias de los principios de «corporate governance» de la OCDE.

Como decimos, este fue también el banderazo de salida, tanto para las empresas asiáticas (incluido Japón, que aprobó su primer código en el año 2001), como para las de Europa central (directamente influidas por el particular proceso de privatización de las empresas públicas que cada una de los Estados utilizó, tras sacudirse el yugo soviético) y las de Sudamérica, en especial Brasil. En casi todos los casos, estos códigos han estado patrocinados y auspiciados por el regulador del mercado de valores de cada uno de los estados. Como muy bien sabemos, en los Estados Unidos, al ser un sistema «ruled based», no se optó por estos códigos de voluntario cumplimiento.

\section{La primera década del siglo. El escándalo «Enron» y la Sarbanes- Oxley Act}

Para el inicio de la siguiente década (año 2000), los inversores institucionales (especialmente los pension funds) habían desarrollado una importante agenda de activismo institucional y comandados por el fondo Calpers ${ }^{66}$, habían conseguido que se despidiese — debido a sus pobres resultados - a los principales ejecutivos y consejeros de grandes sociedades cotizadas como Westinghouse, American Express, IBM, Kodak y General Motors ${ }^{67}$. Así mismo, influyeron de forma importante en el cambio del sistema de retribución de la alta dirección, modificando el «pay for size» por el «pay for performance ${ }^{68}$ ». Este cambio hizo que se dispararan las stock options como modelo de compensación preferido por el management en un mercado eminentemente alcista (bull market), como lo fue el de esa década. No es necesario recordar las lamentables consecuencias que el abuso de estas fórmulas ha ocasionado años después. Los inversores institucionales continuaban presionando, e inclinando poco a poco la balanza de su lado. El management y los consejos de las sociedades, que tan escasa atención habían prestado a

66 California Public Employees' Retirement System: paradigma de fondo activista.

67 JoHn Pound: «The rise of the political model of corporate governance», New York University Law Review, 68, 1993, pp. 1006-59.

68 FRANK DOBBIN y DIRK ZORN, «Corporate malfesance and the myth of shareholders value», Political power \& social theory, 17, 2005, p. 189. En BRIAN R. CHEFFINF, "The history of corporate governance», ECGI Working Papers n. 184 (2012). http:/ssrn.com/abstract-197504. 
los pequeños accionistas; ahora, y ante los nuevos y formidables «vicarios» de los pequeños inversores, empezaban a doblegar la testuz. Sus puestos de trabajo y suculentas remuneraciones estaban en juego.

En 2001 se nombró a Harvey Pitt como nuevo presidente de la SEC, tras la llegada de George W. Bush a la Casa Blanca69. Su estreno en el cargo no pudo ser más accidentado. En efecto, el día 8 de noviembre de 2001 en el modelo 8-K, la empresa Enron ${ }^{70}$, en un inesperado comunicado ${ }^{71}$, informa al mercado de un «restatement» o revisión de sus resultados contables desde el año 1997. Fue, sin duda, el mayor escándalo de la historia bursátil de los Estados Unidos, al menos desde la aprobación de la Securities Exchange Act en 1934. Para Seligman, la pérdida de eficacia de la SEC — debido principalmente a los recortes presupuestarios y al aumento de denuncias por prácticas prohibidas en el mercado- - y el rechazo de la Administración republicana a intervenir en el mercado, causaron un deterioro institucional cuyo máximo exponente fue Enron ${ }^{72}$.

Como resultado de este colapso se creó el «The Enron Special Committee Report», presidido por el decano de la Facultad de Derecho de la Universidad de Texas, William Powers. El resultado de su investigación fue el conocido como «Powers Report ${ }^{73}$ » que en lo que a este trabajo afecta, señala en sus conclusiones dos advertencias directas. La primera, son los evidente fallos en el gobierno corporativo de la sociedad, y la segunda, los errores en la información suministrada al mercado por Enron.

No cabe ninguna duda de que tras este colapso, y debido a la participación activa del gigante de la auditoría Arthur Andersen en la quiebra de Enron (al menos como cooperador necesario), existía un caldo de cultivo propicio a realizar una nueva aproximación a la regulación de los auditores. En enero de 2002, existían tres propuestas de peso en

69 Seligman, The transformation, p. 728

70 Véase John C. Coffee: Gatekeepers. The professions and corporate governance, Oxford University Press, Oxford, 2006. Además de explicar de forma pormenorizada lo que ocurrió con Enron, realiza un análisis realmente interesante sobre el papel desempeñado en esta quiebra por los Gatekeepers. Es decir, por los grupos de profesionales que se relacionan con las empresas, y que en este caso fueron incapaces de detectar ningún problema. En concreto, repasa la actuación de auditores, abogados, analistas y agencias de rating.

71 SEC, http://www.sec.gov/Archives/edgar/data/1024401/000095012901503835/ h91831e8-k.txt.

72 Seligman, The transformation, 728.

73 Georgetown University, http://bodurtha.georgetown.edu/enron/Board_Special_ Report_content.htm. 
relación con la supervisión de los auditores, la tercera de las cuales -la popularmente conocida como la Sarbanes-Oxley Act ${ }^{74}$ - fue aprobada el día 30 de julio. Esta ley crea un Public Company Accounting Oversight Board (PCAOB) que es el organismo que, dependiendo de la SEC, tiene como misión el control de las compañías de auditoría que emiten más de cien informes anualmente.

La Sox indudablemente representa un cambio fundamental en la forma de abordar la publicación de información en los Estados Unidos, ya que desplaza las permisivas normas tradicionales, por normas imperativas. Así mismo, el Gobierno federal está jugando un papel más decisivo desde que la SEC ha extendido sus competencias a áreas que hasta entonces habían sido exclusivamente reguladas por los Estados ${ }^{75}$. Y finalmente, los grupos profesionales «autorregulados ${ }^{76}$ » (contables, auditores y analistas), fueron situados al frente de la discusión sobre gobierno corporativo ${ }^{77}$. Para la ALI, la Sox tiene cinco objetivos básicos: a) reforzar la independencia de las firmas auditoras; b) mejorar la calidad y transparencia de los estados financieros e información societaria; c) mejorar el gobierno corporativo; d) mejorar la objetividad de las investigaciones; e) reforzar la aplicación de las leyes federales, incluyendo la exigencia de responsabilidades penales.

Lo cierto es que si bien la Sox ${ }^{78}$ no realiza una atribución directa de nuevos derechos a los accionistas, ni en términos de derechos de voto,

74 University of Cincinnati, http://www.law.uc.edu/CCL/SOact/soact.pdf.

75 «Sox moved into areas of Corporate Governance historically within the domain of state corporate law». AlAN R. PALMIteR: Securities regulation, Aspen Publisher, New York, 5. ${ }^{\mathrm{a}}$ ed., 2011, p. 38.

76 "Autoregulated», en expressión inglesa. Estos grupos de profesionales, casi no estaban regulados por ninguna norma, al entenderse que era más efectiva la postura de permitir que sus propias organizaciones profesionales fuesen las que aprobaran sus códigos de conducta.

77 GREGORY JACKSON: «Understanding corporate governance in the United States. An historical and theoretical reassessment». Fundación Hans Boeckler, http://www.boeckler.de/pdf/p_arbp_223.pdf, 39.

78 ROBERTA ROMANO: "The Sabarnes-Oxley Act and the making of quack corporate governance», September 25, 2004. NYU, Law and Econ Research Paper 04-032; Yale Law \& Econ Research Paper 297; Yale ICF Working Paper 04-37; ECGI - Finance Working Paper 52/2004. SSRN, http://ssrn.com/abstract=596101 o http://dx.doi.org/10.2139/ ssrn.596101 . La reconocida profesora realiza una crítica devastadora de la ley, por considerarla innecesaria, deficiente técnicamente y cara de implantar para las sociedades; Véase ROBERT CHARLES CLARK: "Corporate governance changes in the wake of the Sarbanes-Oxley Act: a morality tale for policymakers too». http://papers.ssrn.com/sol3/papers.cfm?abstract_id=808244 . Este autor critica la falta de estudios empíricos - de nuevo en la más pura tradición metodológica legal norteamericana- que sustenten las normas promulgadas. 
ni en el de elección de consejeros ${ }^{79}$, indirectamente — tal y como nos dice Talbot ${ }^{80}$ — sus disposiciones sólo les benefician. En el mismo orden de cosas, esta ley apoya decididamente la propuesta de conformar un consejo de administración con más poder y por lo tanto, más independiente del management ${ }^{81}$.

\section{Los últimos esfuerzos regulatorios}

La brutal crisis financiera que sufrió el país desde el año 2007, hizo que se aprobara con carácter de urgencia la denominada Dodd-Frank $\mathrm{Act}^{82}$, que en palabras de la SEC, ha supuesto un:

framework that will support an entirely new regulatory regime designed to bring greater transparency and access to the securities-based swaps market, adopted rules that will result in increased oversight and transparency around hedge fund and other private fund advisers, gave investors a say-on-pay regarding executive compensation and established a whistleblower program which offers incentives for individuals with information regarding securities law violations to come forward. The SEC also has proposed a series of rules designed to improve the practices of credit rating agencies, including rules to limit the conflicts that may arise when NRSROs rely on client payments to drive profits and rules to monitor rating agency employees who move to new positions with rated entities ${ }^{83}$.

Realmente, esta ley sólo afecta al objeto de nuestro trabajo en las siguientes secciones: 1) la sección 951, que es la denominada «say-onpay mandate», que regula la votación periódica (y no vinculante) de los accionistas sobre la retribución del consejo y altos directivos; 2) la sec-

79 Véase LISA M FAIRFAX: «The future of shareholder democracy», Indiana Law Journal, 84, 2009, p. 1259. La autora critica que la SEC no aprobara en el año 2007 un proyecto de norma (a proxy access rule) que obligara a la dirección de las compañías a incluir en las votaciones los candidatos propuestos por accionistas con una determinada representación. Entiende que es un contratiempo importante, y que los accionistas deben continuar su «historical battle for proxy access».

80 LORRAINE E. TALBOT: «Polanyi's embeddedness and shareholder stewardship: a contextual analysis of current anglo-american perspectives on corporate governance», november 18, 2012. NILQ 62(4), 451-68; Symposium paper, Oxford, april 2010; Warwick School of Law Research Paper No. 2012/20. SSRN: http://ssrn.com/ abstract=2177645: 458.

81 BAINBRIDGE, Corporate governance, p. 44.

82 SEC, http://www.sec.gov/about/laws/wallstreetreform-cpa.pdf.

83 SEC, http://www.sec.gov/spotlight/dodd-frank.shtml. 
ción 952, que regula la composición y obligaciones del comité de retribuciones; 3) la sección 953, que permite a la SEC solicitar información adicional, relacionada con la política de retribución; 4) la sección 954, que hace referencia a la devolución de retribuciones a la compañía; 5) la sección 971, que afirma que la SEC tiene autoridad para promulgar una rule sobre la forma en la que cualquier accionista puede utilizar el sistema de delegación de voto para nominar candidatos a un puesto en el consejo y 6) la sección 972, que exige a las compañías informar si la misma persona que es presidente del consejo es, además, máximo ejecutivo de la compañía, y el por qué es, o no, de esta forma ${ }^{84}$.

Como bien sabemos, esta ley es extensísima (848 páginas), y en ella se contienen más de 400 mandatos a las agencias federales, para que regulen determinados aspectos, cien de los cuales se dirigen a la SEC, que tal y como dice el comisionado Gallagher ${ }^{85}$, tiene unos medios escasos.

También ha sido promulgada la denominada Jumpsart Our Business Startups (JOBS) Act, el 3 de enero de 2012, de solo veintidós páginas, en la que tangencialmente, se abordan asuntos relacionados con el gobierno corporativo, al reducirse la información que deben depositar en la SEC aquellas empresas de nueva creación y de dimensiones reducidas. La Administración Obama ha tratado de facilitar la creación y puesta en marcha de pequeñas sociedades, aminorando los costos y cargas impuestas por la SEC y otros organismos, a las grandes empresas. Se flexibiliza tanto la información ${ }^{86}$ que tienen que poner a disposición de público, como los estándares de auditoría87. No obstante, esto ha sido criticado incluso por parte de uno de los cinco miembros del gobierno de la Comisión, para quién esta relajación en las normas relativas a la información que las empresas deben dar al público en general, castigará en última instancia a los inversores ${ }^{88}$.

84 Stephen BaINBRIDGe realiza un furibundo ataque a la ley: «Dodd-Frank is to corporate governance as quackery is to medical practice». "Dodd-Frank: quack federal corporate governance round II», Minnesota Law Review, 95, 2011, pp. 1779-83.

85 SEC, http://www.sec.gov/News/Speech/Detail/Speech/1365171492342\#.Uh3zbG1pCYY. Conferencia dada el 22 de febrero de 2013, en la sede de la SEC, en Washington D.C.

86 Sección 102. SEC, http://www.sec.gov/divisions/corpfin/guidance/cfjjobsactfaqtitle-i-general.htm.

87 Secciones 103 y 104. SEC, http://www.sec.gov/divisions/corpfin/guidance/cfjjobsactfaq-title-i-general.htm.

88 SEC, http://www.sec.gov/News/Speech/Detail/Speech/1365171515808\#.Uh3vEW1pCYY. Conferencia impartida por el comisionado Aguilar el día 19 de abril de 2013, en la Georgia State University, bajo el título «Institutional investors: power and responsibility». 


\section{Conclusiones}

De todo lo expuesto, podemos afirmar que el gobierno corporativo de las sociedades cotizadas en los Estados Unidos, tanto en su vertiente regulada, como en la de voluntario cumplimiento, ha ido evolucionando - madurando, nos atreveríamos a decir - siempre como reacción a una importante crisis. Así es, tanto la Securities Act de 1933, como Securities Exchange Act de 1934, fueron la respuesta legislativa al Crash del 29 y los desmanes de un mercado sin regulación. La crisis del petróleo de los setenta provocó la reacción de la SEC a través de los settlements y los primeros códigos de buen gobierno. Los escándalos del inicio del presente siglo, la Sox, y la crisis de 2008, la Dodd-Frank. Realmente, en la exposición de estos hitos podríamos resumir la evolución del corporate governance.

A través del devenir histórico expuesto, hemos visto como han cambiado las circunstancias socioeconómicas en los Estados Unidos y con ellas, las leyes, la tipología de los accionistas y la forma de interpretar las relaciones de poder en la sociedad mercantil capitalista cotizada. Pero lo que nunca ha cambiado, ha sido la defensa de la idea de que la sociedad debe ser gestionada en primer lugar, en beneficio de sus accionistas.

Son cada vez más los estudios que analizando la historia económico-financiera de los últimos años, empiezan a desvelar el rol jugado por este modelo en la más reciente crisis financiera ${ }^{89}$. Tal vez haya llegado el momento de permitir la entrada de otros constituencies en el órgano de administración de la sociedad, así como de primar la lealtad de los accionistas a largo plazo.

\section{Bibliografía}

AMIHUB, Yacob y BARUCH LEV: "Risk reduction as a managerial motive for conglomerate mergers», Bell Journal of Economics, 12, 1981, pp. 605-617.

AUERBACH, Alan y DAVID ReISHUS: «The impact of taxation on mergers and acquisitions», en Alan AUeRBACH, Mergers and acquisitions, University of Chicago Press, Chicago, 1988.

89 WILLIAM LAZONICK y MARY O'SULLIVAN: "Maximizing shareholder value: a new ideology for corporate governance», Economy \& Society 29, Issue 1, 2000, 13; MICHAEL MARIN: "Disembedding corporate governance: the crisis of shareholder primacy in the UK and Canada», Queen's Law Journal, 39, 2013, p. 223 (totalmente extensible a los EE.UU, de hecho, las referencias son constantes); PAvlos MAsouros: Corporate law and economic stagnation: How Shareholder Value and Short-Termism Contribute to the Decline of the Western Economies, Eleven International, The Hage, 2013. 
BAINBRIDGE, Stephen M.: The new corporate governance in theory and practice, Oxford University Press, New York, 2008.

BalDWIN, Robert, MARTIN CAVE y MaRTIN LODGE, Understanding regulation. Theory, strategy, and practice, Oxford University Press, New York, 2012, 2. ${ }^{\text {a }}$ ed.

CLARK, Robert Charles: "Corporate governance changes in the wake of the Sarbanes-Oxley Act: a morality tale for policymakers too», http://papers. ssrn.com/ sol3/papers.cfm?abstract_id=808244

CoffeE, John C.: Gatekeepers. The professions and corporate governance, Oxford University Press, Oxford, 2006.

DOBBIN, Frank y DIRK ZORN, "Corporate malfesance and the myth of shareholders value», Political power \& social theory, 17, 2005, pp. 179-198.

Dulanvy, Colleen A.: «Social conceptions of the corporation: insights from the history of shareholder voting rights», Washington \& Lee Law Review, 63, 2006, pp. 1347-1388.

EISENBERG, Melvin A.: The structure of the corporation. A legal analysis, Beard Books, Washington, 1976.

FAIRFAX, Lisa M.: "The future of shareholder democracy», Indiana Law Journal, 84, 2009, pp. 1259-1308.

GELTER, Martin: "The pension system and the rise of shareholder primacy», Fordham University School of Law, working paper, june 2012, http://ssrn. com/ abstract=2079607.

GevURTZ, Franklin A.: Corporation law, West, Minnesota, 2010, 2. ${ }^{\text {a ed. }}$

GILSON, Roland J., Myron S. SCHoles y Mark A. Wolfson: «Taxation and the dynamics of corporate control: the uncertain case for tax motivated acquisitions», en JoHn Coffee, LouIs LoWenstein y SUSAn Rose-Ackerman, Knights, riders, and targets: the impact of hostile takeovers, Oxford University Press, New York, 1988.

GIRASA, Roy: Corporate governance \& finance law, Pelgrave-MacMillan, New York, 2013.

GORDON, Jeffrey N.: "The rise of independent directors in the United States, 1950-2005: of shareholder value and stock market prices», Stanford Law Review, 89, 2007, pp. 1435-1568.

HIRSCHMAN, Albert O.: Voice and loyalty: responses to decline in firma, organizations and states, Harvard University Press, Cambridge, Massachusetts, 1970. En CHIU, Iris H-Y.: The foundations and anatomy of shareholder activism, Hart Publishing, Portland, Oregon, 2010.

JENSEN, Michael C.: «Takeovers: their causes and consecuences», Journal of economic Perspectives, 21, 1988.

JACKSON, Gregory: "Understanding corporate governance in the United States. An historical and theoretical reassessment», Fundación Hans Boeckler, http:// www.boeckler.de/pdf/p_arbp_223.pdf.

JENSEN, Michel C. y William H. MECKLING: "Theory of the firm: managerial behavior, agency costs and ownership structure», Journal of Financial Economics, 3, 1976, pp. 305-360.

KAPLAN, Steven N.: "Managements buyouts: evidence on taxes as a source of value», Journal of Finance, 44, 1989. 
LAZONICK, William y Mary O'SULLIVAN: "Maximizing shareholder value: a new ideology for corporate governance», Economy \& Society, 29, Issue 1, 2000, pp. 13-35.

MACEY, Jonathan R.: Corporate governance, Princeton University Press, Princeton, New Jersey, 2008.

- «The transformation of the american law institute», George Washington Law Review, 61, 1993, pp. 1211-1232.

MARENS, Richard: "Inventing corporate governance: the mid-century emergence of shareholder activism», Journal of Business and Management, 8, 2002, 4, pp. 365-390.

MARIN, Michael: «Disembedding corporate governance: the crisis of shareholder primacy in the UK and Canada», Queen's Law Journal, 39, 2013, pp. 223-271.

Masouros, Pavlos: Corporate law and economic stagnation: How Shareholder Value and Short-Termism Contribute to the Decline of the Western Economies, Eleven International, The Hage, 2013.

MORCK, Randall K.: A history of corporate governance around the world: family business groups to professional managers, University of Chicago Press, Chicago, 2005.

MurPHY, Michael E. «The nominating process of corporate boards of directors: a decision making analysis», Berkeley Business Law Journal, 5, 2008, 2, pp. 130-193.

Palmiter, Alan R.: Securities regulation, Aspen Publisher, New York, 2011, 5. a ed.

PORTER, Glenn: The rise of the big business, Wheeling, Wiley-Blackwell, Illinois, 1973, 3. ${ }^{\mathrm{a}} \mathrm{ed}$.

Pound, John: «The rise of the political model of corporate governance», New York University Law Review, 68, 1993, pp. 1003-1071.

Roll, Richard. "The hubris hypothesis of corporate takeovers», Journal of Business, 59, 1986, pp. 197-216.

ROMANEK, Broc: "The pioneers of corporate governance», The Corporate Governance Advisor, 19, 2011, pp. 1-5.

Romano, Roberta: "A guide to takeovers: theory, evidence, and regulation», Yale Journal on Regulation, 9, 1992, pp. 118-180.

- "The Sabarnes-Oxley Act and the making of quack corporate governance» (september 25, 2004). NYU, Law and Econ Research Paper 04-032; Yale Law \& Econ Research Paper 297; Yale ICF Working Paper 04-37; ECGI - Finance Working Paper 52/2004. SSRN, http://ssrn.com/abstract=596101 o http:// dx.doi.org/10.2139/ssrn.596101.

SCHALL, Alexander: "Corporate governance after the dead of the king. The origin of the separation of powers in companies», European Company and Financial Law Review, 8, 2011, 4, pp. 470-495.

SCHIPPER KATHERINE y ABBIE SMITH: «Effects of management buyouts on corporate interest and depreciation tax reductions», Journal of Law and Economic, 34, 1991.

SCHWARTZ, Donald: «Shareholder democracy: a reality or a chimera?», California Management Review, 25, 1983, pp. 53-67. 
Seligman, Joel: The transformation of Wall Street, Aspen Publishers, New York 2003, 3. ${ }^{\text {a }}$ ed.

SOMmer, Alphonse A.: "The impact of the SEC on the corporate governance», Law and Contemporary Problems, 41, 1977, pp. 115-145.

TALBOT, Lorraine: «Polanyi's embeddedness and shareholder stewardship: a contextual analysis of current anglo-american perspectives on corporate governance». (november 18, 2012). NILQ 62(4), pp. 451-68; Symposium paper, Oxford, april 2010; Warwick School of Law Research Paper No. 2012/20. SSRN: http:// ssrn.com/abstract=2177645: pp. 458-490.

Tsuk, Dalia: "Shareholders as proxies: the contours of shareholder democracy», Washington \& Lee Law Review, 2, 2006, pp. 1502-1578.

- «Status bound: the $20^{\text {th }}$ century evolution of directors liability», New York University Journal of Law and Business, 5, 2012, 135.

Wolfson, Mark A.: «Taxation and the dynamics of corporate control: the uncertain case for tax motivated acquisitions», En JoHn COFfEe, LouIs LoWENSTEIN y SUSAN Rose-ACKerman, Knights, riders, and targets: the impact of hostile takeovers, Oxford University Press, New York, 1988. 